\title{
QUANTIFICAÇÃO DE TBHQ (TERC BUTIL HIDROQUINONA) E AVALIAÇÃO DA ESTABILIDADE OXIDATIVA EM ÓLEOS DE GIRASSOL COMERCIAIS
}

Monise Helen Masuchi, Renata Maria dos Santos Celeghini, Lireny Aparecida Guaraldo Gonçalves e Renato Grimaldi* Departamento de Tecnologia de Alimentos, Faculdade de Engenharia de Alimentos, Universidade Estadual de Campinas, CP 6091, 13081-970 Campinas - SP, Brasil

Recebido em 27/3/07; aceito em 11/10/07; publicado na web em 2/4/08

\begin{abstract}
QUANTIFICATION OF TBHQ (TERC BUTYL HYDROQUINONE) AND OXIDATIVE STABILITY EVALUATION OF COMMERCIAL SUNFLOWER OILS. Sunflower oil is one of the vegetable oils most susceptible to oxidative reaction. Therefore the presence of natural (tocopherol) and/or synthetic (TBHQ) antioxidants is a prerequisite to secure the stability of this oil during its shelf life, especially with the increased use of PET packaging. On determining the total tocopherol content in 10 samples of deodorized sunflower oils, high losses in total tocopherol during deodorization were identified. In order to facilitate researche involving oxidative stability of vegetable oils, a method for isolated quantification of TBHQ using the isocratic elution system was developed.
\end{abstract}

Keywords: sunflower oil; oxidative stability; TBHQ.

\section{INTRODUÇÃO}

Óleos vegetais são importantes fontes de energia e de ácidos graxos essenciais (principalmente ácido linoléico - $\omega$-6). Além disso, são importantes também para o funcionamento regular do organismo humano, como veículos no transporte das vitaminas lipossolúveis. ${ }^{1}$

A principal deterioração em óleos é a oxidação. Sabor, aroma e qualidade indesejáveis são algumas das características encontradas em óleos oxidados. ${ }^{1}$

A composição em ácidos graxos, de maneira geral, não se altera durante as etapas do refino (químico ou físico). Porém, as condições de processamento, principalmente durante a desodorização, como é o caso da temperatura e tempo de retenção, alteram a formação dos isômeros trans no produto final. ${ }^{2}$

Sabendo-se que a oxidação de lipídios é uma reação que não pode ser totalmente cessada e que, uma vez iniciada, ocorre em alta velocidade, o seu início pode ser retardado pela adição de antioxidantes. Além disso, para retardar o início da oxidação em óleos vegetais deve-se adotar alguns cuidados no seu processamento, como o uso de equipamentos em aço inox, embalagens herméticas, refrigeração e proteção contra raios ultravioletas. ${ }^{1}$

A estabilidade oxidativa é um dos mais importantes indicadores utilizados para avaliação da qualidade dos óleos comestíveis. ${ }^{3}$ A diferença da estabilidade entre os diversos tipos de óleos vegetais é decorrente principalmente da presença de ácidos graxos poliinsaturados e da quantidade de $\gamma$ - e $\delta$-tocoferóis, além da adição de antioxidantes sintéticos.

Antioxidantes, presentes naturalmente em óleos vegetais ou adicionados a estes, são substâncias inibidoras da oxidação lipídica, ou seja, ajudam na prevenção da deterioração que ocorre em óleos devido à reação de oxidação, causada por espécies reativas de oxigênio. ${ }^{1}$

O principal antioxidante natural encontrado em óleos vegetais é o tocoferol, podendo ocorrer em quatro formas diferentes: $\alpha-, \beta-, \gamma-$ e $\delta$-tocoferol. Esta substância apresenta alto valor nutricional possuindo atividade antioxidante (principalmente nas formas de $\gamma$ - e $\delta$ -

*e-mail: grimaldi@fea.unicamp.br tocoferóis) e poder vitamínico $\mathrm{E}$ (em especial nas formas $\alpha$ - e $\beta$ tocoferóis). ${ }^{4}$

O óleo de girassol, devido ao seu alto teor de ácido linoléico, é um dos óleos vegetais mais susceptíveis à oxidação e, portanto, a presença de antioxidantes é um fator determinante para garantir sua estabilidade oxidativa, principalmente com a crescente utilização das embalagens do tipo PET. Apesar disto, a maioria das empresas deixaram de declarar nas embalagens a presença de TBHQ (terc butil hidroquinona - principal e mais efetivo antioxidante empregado em conjunto com ácido cítrico) sem alterar o prazo de validade dos óleos, o que pode afetar a estabilidade oxidativa dos mesmos e, conseqüentemente, sua vida de prateleira.

Considerando esse contexto, este trabalho teve como finalidade realizar a adaptação da metodologia padrão da $\mathrm{AOCS}^{5}$ para a quantificação de TBHQ, com a utilização do sistema isocrático de eluição ao invés do sistema de gradiente, utilizado para avaliação de mistura de antioxidantes. Assim, com esta modificação, tem-se uma determinação de maior simplicidade, visando facilitar os estudos envolvendo estabilidade oxidativa de óleos vegetais. Além disso, este trabalho também visou avaliar 10 amostras comerciais de óleos de girassol envasados em embalagem PET, principalmente no aspecto da oxidação e de sua composição em ácidos graxos.

\section{PARTE EXPERIMENTAL}

\section{Material}

Foram analisadas 10 amostras de diferentes marcas comerciais de óleo de girassol, envasadas em embalagem PET de $900 \mathrm{~mL}$. A Tabela 1 mostra alguns dados obtidos nos rótulos destas amostras.

Foram ainda utilizadas amostras de óleo de girassol comercial sem a adição de antioxidantes, amostra padrão de TBHQ e solução comercial de TBHQ (20\%) - GRINDOX ${ }^{\mathrm{TM}} 450$.

\section{Métodos}

Ácidos Graxos Livres - Método $\mathrm{AOCS}^{5} \mathrm{Ca} 5 \mathrm{a}-40$ (01); Índice de Peróxido - Método AOCS ${ }^{5}$ Cd 8b-90 (01); Composição em Áci- 
Tabela 1. Informações presentes nos rótulos das amostras de óleo de girassol comerciais

\begin{tabular}{lccc}
\hline Amostras & $\begin{array}{c}\text { Data de } \\
\text { Fabricação }\end{array}$ & $\begin{array}{c}\text { Validade } \\
\text { (meses) }\end{array}$ & $\begin{array}{c}\text { Antioxidante / } \\
\text { Ácido Cítrico }\end{array}$ \\
\hline 1 & $09 / 2005$ & 12 & Não consta \\
2 & $06 / 2005$ & 18 & Não consta \\
3 & $05 / 2005$ & 12 & Ácido cítrico \\
4 & $05 / 2005$ & 12 & Ácido cítrico \\
5 & $06 / 2005$ & 12 & Não consta \\
6 & $09 / 2005$ & 12 & Ácido cítrico \\
7 & $04 / 2005$ & 24 & Não consta \\
8 & $10 / 2005$ & 12 & Ácido cítrico \\
9 & $09 / 2005$ & 18 & Não consta \\
10 & $09 / 2005$ & 12 & Ácido cítrico \\
\hline
\end{tabular}

dos Graxos - Método AOCS 5 Ce 1f-96 (01).

A análise cromatográfica dos ésteres metílicos ${ }^{6}$ foi realizada segundo as seguintes condições: cromatógrafo gasoso capilar CGC Agilent 6850 Series GC System, dotado de coluna capilar DB-23 Agilent (50\% cyanopropyl-methylpoysiloxane), dimensões $60 \mathrm{~m}$, $\phi$ int: $0,25 \mathrm{~mm}, 0,25 \mu \mathrm{m}$ filme. As condições de operação do cromatógrafo foram: fluxo na coluna $=1,00 \mathrm{~mL} / \mathrm{min}$; velocidade linear $=24 \mathrm{~cm} / \mathrm{s}$; temperatura do detector: $280{ }^{\circ} \mathrm{C}$; temperatura do injetor: $250{ }^{\circ} \mathrm{C}$; temperatura do forno: $110{ }^{\circ} \mathrm{C}-5 \mathrm{~min}, 110-215$ ${ }^{\circ} \mathrm{C}\left(5{ }^{\circ} \mathrm{C} / \mathrm{min}\right), 215^{\circ} \mathrm{C}-24 \mathrm{~min}$; gás de arraste: hélio; volume injetado: $1,0 \mu \mathrm{L}$.

Estabilidade Oxidativa - Método AOCS ${ }^{5}$ Cd 12b-92 (01)

Equipamento utilizado: oxidative stability instrument - Omnion. As condições da análise foram: temperatura: $110{ }^{\circ} \mathrm{C} ; 5 \mathrm{~g}$ de amostra e $9,0 \mathrm{~L}$ ar/h.

Tocoferóis - cromatografia líquida de alta eficiência (CLAE) Método $\mathrm{AOCS}^{5}$ Ce 8-89 (01)

Equipamento e condições da análise: bomba isocrática Perkin Elmer 200; detector fluorescência LC 240 Perkin Elmer, excitação $290 \mathrm{~nm}$, emissão $330 \mathrm{~nm}$; fluxo de $1,0 \mathrm{~mL} / \mathrm{min}$ coluna analítica (Merck) 250 x 4 mm Li Chrosorb Si 60* $5 \mu \mathrm{m}$, acoplada à coluna de guarda compatível; fase móvel: hexano/isopropanol 99:1 (solventes grau HPLC filtrados e degaseificados por $10 \mathrm{~min}$ em banho ultrasom). A quantificação foi realizada através de curva de padronização externa dos quatro tocoferóis e de padrões diários de referência.

TBHQ - cromatografia líquida de alta eficiência (CLAE) - Adequação do Método $\mathrm{AOCS}^{5} \mathrm{Ce}$ 6-86 (01)

Equipamento e condições da análise: sistema isocrático FCV - 10 AL VP acoplado ao forno de coluna CTO - 10AS VP Shimadzu (Temperatura do forno $=30{ }^{\circ} \mathrm{C}$ ); detector UV/Vis SPD - 10A VP, $280 \mathrm{~nm}$; controle de sistema SCL - 10A VP; fase móvel: água/acetonitrila 70:30, v/v (solvente grau HPLC filtrado e água Milli-Q); fluxo: 1,0 mL/min; coluna fase reversa C18 $\mu$-Bondapack (300 mm x 3,9 mm x $10 \mu \mathrm{m}$ ); extração do antioxidante TBHQ com hexano e acetonitrila, de acordo com a metodologia oficial citada. A quantificação foi realizada através de curva de padronização externa.

\section{RESULTADOS E DISCUSSÃO}

Os valores máximos permitidos para ácidos graxos livres e índice de peróxido nos óleos vegetais refinados, segundo a Resolução ANVISA $^{7}$ RDC n ${ }^{\circ} 270$, de 22 de setembro de 2005, são de $0,3 \%$ em ácido oléico e $10 \mathrm{meq} \mathrm{O}_{2} / \mathrm{kg}$, respectivamente, que não cita se os mesmos devem estar de acordo com esta resolução no final da sua validade declarada. A Tabela 2 mostra os valores de ácidos graxos livres e de índice de peróxido determinados para as
Tabela 2. Ácidos graxos livres (AGL em \% ácido oléico), índice de peróxido (IP em meq $\mathrm{O}_{2} / \mathrm{Kg}$ ) e tempo (t em meses) transcorrido a partir da fabricação até a realização das análises em amostras de óleo de girassol comerciais

\begin{tabular}{lcccccc}
\hline Amostras & $\mathrm{t}_{1}$ & $\mathrm{AGL}_{1}$ & $\mathrm{IP}_{1}$ & $\mathrm{t}_{2}$ & $\mathrm{AGL}_{2}$ & $\mathrm{IP}_{2}$ \\
\hline 1 & 1 & 0,07 & 2,8 & 10 & 0,05 & 4,0 \\
2 & 4 & 0,14 & 10,8 & 13 & 0,12 & 6,8 \\
3 & 5 & 0,10 & 9,1 & 11,5 & 0,08 & 4,1 \\
4 & 5 & 0,11 & 8,3 & 11,5 & 0,08 & 3,6 \\
5 & 4 & 0,09 & 13,3 & 11 & 0,06 & 5,7 \\
6 & 1 & 0,06 & 2,1 & 10 & 0,03 & 5,5 \\
7 & 6 & 0,30 & 10,8 & 15 & 0,41 & 6,4 \\
8 & 1 & 0,05 & 5,3 & 10 & 0,04 & 5,3 \\
9 & 1 & 0,07 & 8,4 & 10 & 0,30 & 3,6 \\
10 & 1 & 0,06 & 6,6 & 10 & 0,03 & 5,4 \\
\hline
\end{tabular}

dez amostras de óleo de girassol comerciais em dois tempos transcorridos a partir de sua data de fabricação. Segundo a metodologia oficial da AOCS, ${ }^{5}$ o coeficiente de variação para as análises de ácidos graxos livres e índice de peróxido em óleos refinados é de, aproximadamente, $10 \%$.

A grande variabilidade dos valores de índice de peróxido pode ser explicada pelo fato de que esta análise mede apenas os compostos primários de oxidação e que possui a tendência de queda, quando ocorre o aparecimento de compostos secundários, que podem ser determinados por outros índices. Dentre eles, pode-se citar o índice de anisidina que, além de não possuir legislação específica, apresenta alta complexidade de execução e é pouco utilizado como análise de rotina. ${ }^{8}$

Entre as amostras analisadas observa-se que a de $n^{\circ} 7$ apresentou teor de ácidos graxos livres além do limite máximo de 0,3\% estabelecido pela lei vigente. Quanto ao índice de peróxido, verifica-se a ocorrência de valores maiores que o estipulado pela ANVISA $^{7}$ nos índices das amostras 2, 5 e 7, porém, considerando o coeficiente de variação de $10 \%$ para esta análise, apenas a amostra 5 não se enquadra no limite da legislação.

Apesar da composição em ácidos graxos ser uma identidade específica e de pouca variabilidade nos óleos vegetais, esta afirmação não se aplica completamente no caso do óleo de girassol, uma vez que existem no mercado mundial variedades submetidas a melhoramentos genéticos, basicamente visando a redução do ácido linoléico e o conseqüente aumento do ácido oléico. ${ }^{8}$ As Tabelas 3 e 4 mostram a composição em ácidos graxos das amostras comerciais de óleo de girassol.

Conforme esperado, ocorreu grande variabilidade nos teores de ácidos oléico e linoléico, observando-se valores de 18 a $28 \%$ e de 59 a $69 \%$, respectivamente. Porém, não foram encontradas amostras de óleo de girassol alto oléico, o que seria desejável para aumentar a estabilidade oxidativa. ${ }^{8}$

Desde o final de julho de 2006, todos os alimentos industrializados passaram a ter a obrigação da declaração do teor de isômeros trans na sua rotulagem. No caso do óleo de girassol, o teor de isômeros trans formado após o refino físico é ao redor de 2,56\%, enquanto que após o refino químico este teor é cerca de $0,76 \% .^{2}$

Os valores encontrados variaram de 0,19 a 1,96\% de isômeros trans. De acordo com a Resolução ANVISA ${ }^{9}$ RDC n ${ }^{\circ} 360$, de 23 de dezembro de 2003, pode-se considerar como "zero" ou "não contém" trans valores de até $0,2 \mathrm{~g}$ trans/porção de alimento, sendo neste caso considerada a porção de $13 \mathrm{~mL}$. Apenas a amostra 9, com $1,96 \%$ de isômeros trans, teria que ser rotulada com o valor impresso de aproximadamente $0,25 \mathrm{~g}$ trans $/ 13 \mathrm{~mL}$. 
Tabela 3. Composição (\%) em ácidos graxos das amostras 1 a 5 de óleo de girassol comerciais

\begin{tabular}{lccccc}
\hline & \multicolumn{5}{c}{ Amostras } \\
Ácidos Graxos (\%) & 1 & 2 & 3 & 4 & 5 \\
\hline C 14:0 & 0,1 & 0,1 & 0,1 & 0,1 & 0,1 \\
C 16:0 & 6,2 & 6,5 & 6,7 & 6,7 & 6,2 \\
C 16:1 & 0,1 & 0,1 & - & - & - \\
C 18:0 & 3,5 & 3,6 & 3,8 & 3,9 & 3,5 \\
C 18:1 (trans) & - & - & - & - & - \\
C 18:1 (cis) & 26,2 & 26,2 & 17,6 & 17,6 & 25,4 \\
C 18:2 (trans) & 0,2 & 0,8 & 0,5 & 0,5 & 0,4 \\
C 18:2 (cis) & 62,0 & 60,9 & 69,3 & 69,3 & 62,8 \\
C 18:3 (trans) & - & - & - & - & - \\
C 18:3 (cis) & 0,4 & 0,4 & 0,2 & 0,2 & 0,2 \\
C 20:0 & 0,3 & 0,3 & 0,3 & 0,3 & 0,2 \\
C 20:1 & 0,2 & 0,3 & 0,3 & 0,3 & 0,2 \\
C 22:0 & 0,7 & 0,7 & 0,8 & 0,8 & 0,7 \\
C 22:1 & - & - & 0,1 & 0,1 & - \\
C 24:0 & 0,2 & 0,2 & 0,3 & 0,3 & 0,2 \\
\hline Saturados & 10,9 & 11,3 & 11,9 & 12 & 10,9 \\
Monoinsaturados & 26,6 & 26,6 & 18,0 & 18,0 & 25,7 \\
Polinsaturados & 62,5 & 62,1 & 70,1 & 70,1 & 63,4 \\
Isômeros trans & 0,19 & 0,84 & 0,52 & 0,52 & 0,39 \\
\hline
\end{tabular}

Tabela 4. Composição (\%) em ácidos graxos das amostras 6 a 10 de óleo de girassol comerciais

\begin{tabular}{lccccc}
\hline & \multicolumn{5}{c}{ Amostras } \\
Ácidos Graxos $(\%)$ & 6 & 7 & 8 & 9 & 10 \\
\hline C 14:0 & 0,1 & 0,1 & 0,1 & 0,1 & 0,1 \\
C 16:0 & 6,6 & 6,0 & 6,6 & 7,6 & 6,9 \\
C 16:1 & - & - & - & 0,1 & - \\
C 18:0 & 3,6 & 3,5 & 3,5 & 3,5 & 3,2 \\
C 18:1 (trans) & - & - & - & - & - \\
C 18:1 (cis) & 19,1 & 27,9 & 20,2 & 24,9 & 19,6 \\
C 18:2 (trans) & 0,3 & 0,2 & 0,4 & 1,2 & 0,3 \\
C 18:2 (cis) & 68,5 & 60,9 & 67,5 & 59,2 & 68,1 \\
C 18:3 (trans) & - & - & - & 0,8 & - \\
C 18:3 (cis) & 0,2 & - & 0,3 & 0,9 & 0,6 \\
C 20:0 & 0,3 & 0,2 & 0,3 & 0,3 & 0,2 \\
C 20:1 & 0,2 & 0,2 & 0,3 & 0,4 & 0,2 \\
C 22:0 & 0,7 & 0,7 & 0,7 & 0,7 & 0,6 \\
C 22:1 & - & - & - & - & - \\
C 24:0 & 0,3 & 0,2 & 0,3 & 0,3 & 0,2 \\
\hline Saturados & 11,6 & 10,7 & 11,5 & 12,5 & 11,2 \\
Monoinsaturados & 19,3 & 28,1 & 20,5 & 25,4 & 19,7 \\
Polinsaturados & 69,1 & 61,2 & 68,1 & 62,1 & 69,0 \\
Isômeros trans & 0,32 & 0,22 & 0,35 & 1,96 & 0,28 \\
\hline
\end{tabular}

Sabendo-se que o refino do óleo de girassol é sempre químico, pode-se concluir que as diferenças encontradas entre as amostras quanto ao teor de isômeros trans são decorrentes de alterações nas condições do processo, em especial na etapa de desodorização. ${ }^{2}$

A formação de ácidos graxos trans no óleo refinado ocorre praticamente na desodorização, sendo temperatura elevada e tempo de retenção prolongado os principais fatores. Segundo O’Brien, ${ }^{8}$ a formação de isômeros trans durante a desodorização começa a ser significante quando realizada entre 220 e $240{ }^{\circ} \mathrm{C}$ e aumenta quase exponencialmente a partir de $240{ }^{\circ} \mathrm{C}$. Já um tempo de retenção prolongado depende do sistema de desodorização utilizado. Em sistemas descontínuos, um período de retenção adequado para minimizar a formação de isômeros trans varia entre 3 a 8 h, enquanto que em sistemas contínuos e semi-contínuos este tempo varia entre 15 a $120 \mathrm{~min}$, dependendo da temperatura do processo. ${ }^{8}$

O maior teor de isômeros trans foi encontrado na amostra 9 $(1,96 \%)$, podendo caracterizar a utilização de temperaturas acima de $240{ }^{\circ} \mathrm{C}$ ou mesmo tempo de retenção prolongado na sua etapa de desodorização. ${ }^{2}$

Existe uma recomendação internacional para que se diminuam as temperaturas de desodorização, de modo a atender aos padrões de qualidade internacionais que são, na maioria deles, inferiores a $1 \%$ de isômeros trans. ${ }^{2}$ Este padrão de qualidade internacional existe devido ao fato de que o alto consumo de ácidos graxos trans, assim como de ácidos graxos saturados, aumenta o risco de doenças cardiovasculares. $^{10}$

As condições de processamento dos óleos vegetais afetam diretamente o teor de tocoferóis, os quais podem ter importância fundamental na estabilidade oxidativa deste óleo, mesmo sendo um componente minoritário.

Segundo o Codex Alimentarius, ${ }^{11}$ o teor de tocoferóis totais para o óleo de girassol bruto varia entre 440 a $1520 \mathrm{mg} / \mathrm{kg}$, porém para uma estimativa de perda durante o processo, optou-se pela variação relatada por Alpaslan et al, ${ }^{12}$ que está entre 755 a $855 \mathrm{mg} / \mathrm{kg}$. A Tabela 5 mostra o teor de tocoferol total $(\mathrm{mg} / \mathrm{kg})$ e a perda estimada $(\%)$ de tocoferol apresentadas pelas amostras de óleo desodorizado, considerando que o óleo bruto possui, em média, $820 \mathrm{mg} / \mathrm{kg}$ de tocoferóis totais. ${ }^{12}$

Tabela 5. Teor de tocoferol total (mg/kg) e perda estimada (\%) de tocoferol em relação ao óleo bruto em amostras de óleo de girassol comerciais

\begin{tabular}{lcc}
\hline Amostras & Tocoferol Total & Perda Estimada \\
\hline 1 & 670,3 & 18 \\
2 & 625,6 & 23 \\
3 & 747,3 & 9 \\
4 & 755,9 & 8 \\
5 & 743,9 & 9 \\
6 & 700,6 & 14 \\
7 & 521,7 & 36 \\
8 & 581,0 & 29 \\
9 & 404,9 & 50 \\
10 & 588,6 & 28 \\
\hline
\end{tabular}

O teor de tocoferóis em óleos vegetais decresce durante o refino, principalmente na etapa de desodorização na qual o óleo é submetido à alta temperatura por um determinado tempo. Dependendo das condições do processamento, a perda do tocoferol pode ser de até $30 \%$ comparando-se ao valor encontrado no óleo bruto. Assim, observa-se que a maioria das amostras analisadas está dentro desta estimativa, exceto as amostras 7 e 9, que possivelmente apresentaram excessivas perdas de tocoferóis totais, apesar de não ser conhecido o valor real de tocoferol dos óleos brutos das amostras analisadas. ${ }^{4}$

Os refinos físico e químico podem ocasionar maiores perdas de tocoferol se a temperatura de desodorização usada for superior a $260{ }^{\circ} \mathrm{C}$. Assim, comparando-se a amostra 9 com as demais, observa-se que seu baixo teor de tocoferóis totais $(404,9 \mathrm{mg} / \mathrm{kg}$ correspondente a uma perda estimada de 50\%) se deve, provavelmente, ao uso de elevadas temperaturas na etapa de desodorização, fato anteriormente observado na composição em ácidos graxos e que possivelmente levou à formação de alto teor de isômeros trans. ${ }^{8}$

A oxidação é a principal causa da degradação dos óleos vege- 
Tabela 6. Estabilidade oxidativa (OSI em $\left.\mathrm{h}-110^{\circ} \mathrm{C}\right)$ e estabilidade AOM (calculada pelo equipamento em $\mathrm{h}-97,8{ }^{\circ} \mathrm{C}$ ) em amostras de óleo de girassol comerciais

\begin{tabular}{lcc}
\hline Amostras & OSI & AOM \\
\hline 1 & 6,0 & 14,1 \\
2 & 4,8 & 11,2 \\
3 & 3,9 & 9,0 \\
4 & 3,8 & 8,7 \\
5 & 4,8 & 11,2 \\
6 & 5,7 & 13,3 \\
7 & 4,3 & 9,9 \\
8 & 5,7 & 13,3 \\
9 & 5,4 & 12,6 \\
10 & 6,3 & 14,8 \\
\hline
\end{tabular}

tais, sendo a estabilidade oxidativa um dos principais indicadores desta deterioração, além de ser um importante parâmetro utilizado para se determinar a vida de prateleira de produtos contendo óleos e/ou gorduras. A Tabela 6 mostra o valor da estabilidade oxidativa (OSI) a $110{ }^{\circ} \mathrm{C}$ e da estabilidade AOM (Active Oxygen Method) das amostras de óleo de girassol comerciais.

O Índice de Estabilidade Oxidativa (OSI) é uma análise automatizada do Método do Oxigênio Ativo (AOM). Enquanto que no método AOM se determina o tempo em horas necessário para que o óleo alcance um valor de índice de peróxido igual a $100 \mathrm{meq}$ $\mathrm{O}_{2} / \mathrm{kg}$, o método OSI mede a variação da condutividade da água, quando são formados os compostos de oxidação. O período ou tempo de indução é o tempo em horas que o óleo resiste antes do aumento brusco da condutividade. Como não foram encontrados dados na literatura para estabilidade oxidativa (OSI) para o óleo de girassol típico, a correlação entre os métodos foi realizada através da programação do equipamento utilizado neste trabalho (oxidative stability instrument - Omnion).

A estabilidade AOM do óleo de girassol típico deve ser de, no mínimo, 10 h. Assim, se comparadas ao óleo de girassol típico, as amostras 3 e 4 apresentam baixa estabilidade oxidativa, que pode ser devida a vários fatores, todos relacionados ao processamento. ${ }^{8}$

A estabilidade oxidativa dos óleos está relacionada ao grau de insaturação e à presença de antioxidantes naturais (tocoferóis) ou sintéticos. Desta forma, pode-se inferir que a menor estabilidade oxidativa do óleo de girassol, quando comparada ao óleo de soja, é devida ao baixo teor de $\gamma$ - e $\delta$-tocoferóis. ${ }^{4}$ Essa diferença existente entre as quantidades de $\gamma$ - e $\delta$-tocoferóis entre esses dois óleos pode ser observada no trabalho de Warner, ${ }^{13}$ em que o óleo de soja apresentou 610 e $260 \mathrm{mg} / \mathrm{kg}$ destes dois tocoferóis, respectivamente, enquanto que no óleo de girassol os valores foram de 30 e $10 \mathrm{mg} /$ $\mathrm{kg}$, respectivamente.

Diferenças na estabilidade oxidativa entre óleos também podem ser atribuídas ao grau de insaturação dos ácidos graxos. ${ }^{14} \mathrm{~A}$ presença de elevados teores de ácido linoléico, altamente susceptível à oxidação, acarreta no aumento da velocidade da reação de oxidação, diminuindo sua estabilidade oxidativa. ${ }^{15}$

Com o objetivo de facilitar estudos envolvendo estabilidade oxidativa em óleos vegetais, adaptou-se a metodologia oficial da $\operatorname{AOCS}^{5}$ (Ce 6-86), que utiliza sistema gradiente de eluição para determinação simultânea de antioxidantes sintéticos - PG, THBP, TBHQ, NDGA, BHA, Ionox-100 e BHT - através da cromatografia líquida de alta eficiência (CLAE).

Sabendo-se que o antioxidante mais utilizado em óleos vegetais é o TBHQ, realizou-se a modificação do método anteriormente citado para a determinação isolada deste antioxidante. Para isto, substituiu-se o sistema gradiente de eluição pelo isocrático, que apresenta maior simplicidade.

Após a realização de testes com diferentes solventes e concentrações, determinou-se que a fase móvel mais eficiente na determinação de TBHQ é a formada por água/acetonitrila (70:30, v/v). As demais condições cromatográficas utilizadas foram citadas anteriormente na Parte Experimental. A Figura 1 mostra o perfil cromatográfico (CLAE) de amostra padrão de TBHQ $(100 \mu \mathrm{g} / \mathrm{mL})$, obtido através da aplicação do método modificado.

Os limites de detecção e de quantificação, nas condições utilizadas neste trabalho, foram 1,0 e 2,0 $\mu \mathrm{g} / \mathrm{mL}$, respectivamente.

Para determinar o fator de recuperação do TBHQ, foram utilizadas amostras de óleo de girassol puro - sem a adição prévia de antioxidantes - e solução comercial de TBHQ, obtendo-se amostras em três concentrações diferentes - 50, 100 e 150 mg de TBHQ/ $\mathrm{kg}$ de óleo de girassol.

De acordo com o método oficial da AOCS, ${ }^{5}$ realizou-se a etapa de extração do antioxidante com hexano e acetonitrila e foram quantificadas as concentrações de TBHQ, utilizando o método adaptado.

Os fatores de recuperação encontrados para os três níveis de concentrações - 50, 100 e $150 \mathrm{mg} / \mathrm{kg}$ - foram de 80,80 e $88 \%$, respectivamente. Assim, tem-se que o valor final do fator de recuperação é da ordem de $80 \%$ para as condições utilizadas nesta análise, valor que pode ser considerado baixo. O método oficial da $\mathrm{AOCS}^{5}$ determinou um fator de recuperação de $98 \%$ para o TBHQ, o que não foi alcançado neste trabalho, apesar de todas as precauções citadas no método terem sido seguidas.

Ao aplicar o método modificado de determinação de TBHQ nas 10 amostras de óleo de girassol comerciais, analisadas neste trabalho, constatou-se que nenhuma delas apresentou em sua composição o antioxidante TBHQ, o que condiz com seus rótulos, que não mencionam a adição de tal composto.

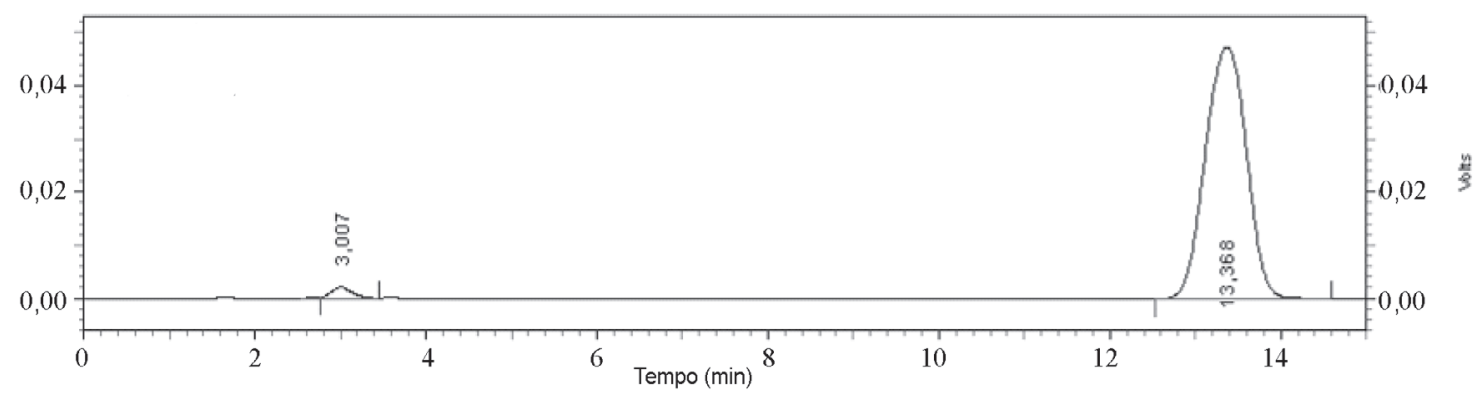

Figura 1. Exemplo de perfil cromatográfico obtido da análise por cromatografia líquida de alta eficiência (CLAE) da amostra padrão de TBHQ com concentração de $100 \mu \mathrm{g} / \mathrm{mL}$ 
O TBHQ apresenta maior custo que outros antioxidantes. Este pode ser um motivo pelo qual não se tem mais adicionado TBHQ a óleos de girassol comerciais. Outro motivo para a não utilização de TBHQ nos óleos analisados pode estar relacionado com sua grande rotatividade no mercado. ${ }^{16}$

\section{CONCLUSÃO}

Ao analisar os dados obtidos para a estabilidade oxidativa das dez amostras de óleo de girassol comerciais, constatou-se que apenas duas amostras ( 3 e 4) apresentaram valores de estabilidade AOM abaixo do recomendado na literatura.

Apenas 5 entre as 10 amostras obedeceram a todas recomendações de estabilidade e qualidade, incluindo os limites da legislação brasileira.

Não foram encontradas variedades com alto teor de ácido oléico.

Quanto à adequação da metodologia para quantificação do TBHQ com sistema isocrático, foi obtido resultado satisfatório, sendo esta análise plenamente viável para quando não houver mistura de antioxidantes. A maior dificuldade encontrada foi na etapa de extração, o que justifica seu fator de recuperação médio de $80 \%$.

Conforme declarado nos rótulos, nenhuma marca analisada está utilizando TBHQ nos produtos.

\section{AGRADECIMENTOS}

À FAPESP (Fundação de Amparo à Pesquisa do Estado de São Paulo), financiadora da bolsa de Iniciação Científica.

\section{REFERÊNCIAS}

1. Lindley, M. G.; Trends Food Sci. Technol. 1998, 9, 336.

2. Tasan, M.; Demirci, M.; J. Am. Oil Chem. Soc. 2003, 80, 825

3. Tan, C. P.; Che Man, Y. B.; Selamat, J.; Yusoff, M. S. A.; Food Chem. 2002, 76,385 .

4. Tasan, M.; Demirci, M.; Eur. Food Res. Technol. 2005, 220, 251.

5. AOCS - American Oil Chemists Society; Official Methods and Recommended Pratices of the American Oil Chemists' Society, $5^{\text {th }}$ ed., Washington, 2001.

6. Hartman, L.; Lago, R.; Lab. Practice 1973, 22, 475.

7. ANVISA - Agência Nacional da Vigilância Sanitária; RDC no 270, de 22 de setembro de 2005 - Regulamento técnico para óleos vegetais, gorduras vegetais e creme vegetal.

8. O’Brien, R. D.; Formulating and Processing for Applications. Fats and Oils, $2^{\text {nd }}$ ed., CRC Press, 2004.

9. ANVISA - Agência Nacional da Vigilância Sanitária; RDC n 360, de 23 de dezembro de 2003 - Regulamento técnico sobre rotulagem nutricional de alimentos embalados.

10. Mason, P.; Pharm. J. 2004, 273, 750 .

11. Codex Alimentarius (FAO/WHO), Codex Standard for Named Vegetable Oils, CODEX STAN 210 (Amended 2003), Codex Alimentarius, Roma, Itália, 2003.

12. Alpaslan, M.; Tepe, S.; Simsek, O.; Int. J. Food Sci. Technol. 2001, 36, 737.

13. Warner, K.; J. Agric. Food Chem. 2005, 53, 9906

14. Martín-Povillo, M.; Márquez-Ruiz, G.; Dobarganes, M. C.; J. Am. Oil Chem. Soc. 2004, 81, 577.

15. Crapiste, G. H.; Brevedan, M. I. V.; Carelli, A. A.; J. Am. Oil Chem. Soc. 1999, 77, 1437.

16. Campos, G. C. M.; Toledo, M. C. F.; Braz. J. Food Technol. 2000, 3, 65. 